\title{
ENTIRE FUNCTIONS WITH FINE ASYMPTOTIC ESTIMATES FOR CONVEX FUNCTIONS
}

\author{
K.P. ISAEV, R.S. YULMUKHAMETOV, A.A. YUNUSOV
}

\begin{abstract}
In the paper we propose an entire function such that the logarithm of its modulus asymptotically approximates the given subharmonic function $\widetilde{h}(\operatorname{Re} z)$, where $\widetilde{h}$ is the Legendre transformation of a convex function $h(t)$ on $(-1 ; 1)$. Such functions have applications in the issues on representation by exponential series of functions in integral weighted spaces on the interval $(-1 ; 1)$ with the weight $\exp h(t)$. At that, better the approximation, a finer topology can be used for the representation by exponential series. For functions $h$ obeying $(1-|t|)^{n}=O(\exp (h(t))), n \in \mathbb{N}$, the corresponding entire functions were constructed before. In the present paper we consider the functions satisfying $\exp (h(t))=o\left((1-|t|)^{n}\right), n \in \mathbb{N}$. In the suggested construction we take into consideration the necessary conditions for the distribution of exponents for the exponentials in the unconditional bases obtained in previous works. This is why the main result of the paper (Theorem 1) should be treated not as a tool for constructing unconditional bases but as an argument supporting the absence of such bases.
\end{abstract}

Keywords: entire functions, subharmonic function, Riesz measure, Hilbert space, Riesz bases.

Mathematics Subject Classification: 30D20

\section{INTRODUCTION}

The problem on approximating subharmonic functions by the logarithm of the modulus of an analytic function appeared in the theory of entire functions. The first general result for this problem was proven in work [1]. In [2] there was proven the following essential specification of V.S. Azarin's theorem.

Theorem A. For each subharmonic on the plane function u of finite growth there exists an entire function $f$ satisfying condition

$$
|u(z)-\ln | f(z)||=O(\ln |z|), \quad z \notin E, \quad|z| \longrightarrow \infty .
$$

The exceptional set is small, for instance, it has a finite Lebesgue measure.

This theorem is the best possible and optimal in the sense of the estimate for the difference and the size of the exceptional set. However, while applying such results in the related issues of complex analysis one needs finer estimates for the difference. Introducing additional conditions for function $u$ and increasing exceptional set $E$, in [3]- 5] authors succeeded to obtain finer estimates for the difference. In the latter work there were considered the functions $\widetilde{h}(z)=$ $\widetilde{h}(\operatorname{Re} z)$ being the Legendre transform of a convex function $h(t)$ defined on the interval $(-1 ; 1)$ of the real axis. The approximation of such functions has applications in issues on representing

K.P. Isaev, R.S. Yulmukhametov, A.A. Yunusov, Entire functions with fine asymptotic esTIMATES FOR CONVEX FUNCTIONS.

(c) Isaev K.P., Yulmukhametov R.S., Yunusov A.A. 2014.

Submitted February 22, 2014. 
functions in integral weighter Hilbert spaces on intervals by the exponentials series. In work [5] there were constructed entire functions quite well approximating convex functions of the mentioned type, if $e^{h(t)}(1-|t|)^{n} \longrightarrow \infty$ for each $n$ as $|t| \longrightarrow 1$.

The present paper is devoted to constructing an entire function approximating asymptotically functions $\widetilde{h}$ when $e^{h(t)}(1-|t|)^{n}=o(1)$ for each $n$ as $|t| \longrightarrow 1$.

Construction of entire function. Let $u(x)$ be a non-negative twice differentiable convex function on $\mathbb{R}, u(0)=0,|x| u^{\prime \prime}(x)$ decays as $|x|$ increases and

$$
u^{\prime \prime}(x)=o\left(1 /|x|^{2}\right),|x| \longrightarrow \infty .
$$

We define two increasing sequences $T_{n}$ and $x_{n} \in\left(T_{n}, T_{n+1}\right), n \in \mathbb{Z}$, by the relations

$$
T_{0}=0, \quad\left(T_{n+1}-T_{n}\right) \int_{T_{n}}^{T_{n+1}} d u^{\prime}(x)=1, \quad n \in \mathbb{Z}, \quad n \neq 0, \quad \int_{T_{n}}^{T_{n+1}}\left(x-x_{n}\right) d u^{\prime}(x)=0 .
$$

We let $p_{n}=T_{n+1}-T_{n}$. The squares

$$
P_{k, m}=\left\{z=x+i y: T_{k}<x<T_{k+1},\left|y-p_{k}\left(m+\frac{1}{2}\right)\right|<\frac{p_{k}}{2}\right\}
$$

are mutually disjoint and the points $w_{k, m}=x_{k}+i\left(m+\frac{1}{2}\right), k, m \in \mathbb{Z}$, are the mass centers of these squares w.r.t. the measure $d u^{\prime}(x) d y$. For $q \in(0 ; 1)$ we let

$$
P_{k, n}^{q}=w_{k, n}+q\left(P_{k, n}-w_{k, n}\right) .
$$

Theorem 1. There exists an entire function $f(z)$ with simple zeroes $w_{k, m}=x_{k}+i p_{k}\left(m+\frac{1}{2}\right)$, $k, m \in \mathbb{Z}$, obeying the conditions

1. For each $z \in \mathbb{C}$ the estimate

$$
\ln |f(z)| \leqslant u(\operatorname{Re} z)+O(N(2|z|)), \quad|z| \longrightarrow \infty,
$$

holds true, where $N(r)$ is the counting function of the sequence $\left|x_{k}\right|$ :

$$
N(r)=\sum_{\left|x_{k}\right| \leqslant r} 1
$$

2. For $z \notin \bigcup_{k, n} P_{k, n}^{q}$ the estimate

$$
\ln |f(z)| \geqslant u(\operatorname{Re} z)-2 \ln |z|+O(N(2|z|)), \quad|z|>1, \quad|z| \longrightarrow \infty,
$$

holds true.

Proof of Theorem 1. We can assume that $u(0)=u^{\prime}(0)=0$. We begin with a preliminary lemma.

Lemma 1. For the piece-wise linear convex function $v(x)$ on $\mathbb{R}$ defined by the condition that its derivative is a piece-wise constant function with the discontinuity points $\left\{x_{n}\right\}$ and the jump $p_{n}$ at point $x_{n}$ and $v(0)=v^{\prime}(0)=0$ the condition

$$
\sup _{x \in \mathbb{R}}|v(x)-u(x)|<1
$$

is satisfied. Moreover, the conditions for function $u$ implies that sequence $p_{n}$ increases as $|n| \longrightarrow \infty$ and

$$
\begin{gathered}
\underline{\lim }_{n \rightarrow \infty} \frac{T_{n+1}}{T_{n}}=\infty, \quad \underline{\lim }_{n \longrightarrow-\infty} \frac{T_{n}}{T_{n+1}}=\infty \\
0 \leqslant T_{n}<x_{n} \leqslant \frac{T_{n+1}+T_{n}}{2}, \quad n \geqslant 0, \quad 0>T_{n+1}>x_{n}>\frac{T_{n+1}+T_{n}}{2}, \quad n<0 .
\end{gathered}
$$


Proof. By the induction in $n$ one can prove that for each $n$ one has $u\left(T_{n}\right)=v\left(T_{n}\right), u^{\prime}\left(T_{n}\right)=$ $v^{\prime}\left(T_{n}\right)$. Therefore, function $v(x)$ is the upper envelope for the tangent functions of $u(x)$ at points $T_{n}$ and

$$
\sup _{x \in \mathbb{R}}|u(x)-v(x)|=\sup _{n \in \mathbb{Z}}\left(u\left(x_{n}\right)-v\left(x_{n}\right)\right) .
$$

The estimate of the latter supremum can be done by elementary methods.

Due to this lemma we can prove the theorem assuming that $u$ is a piece-wise linear function. By $\mu$ we denote the measure associated with the subharmonic function $u(z)=u(\operatorname{Re} z)$. We observe immediately that measure $\mu$ is concentrated on the vertical lines $\operatorname{Re} z=x_{k}$ and on each of these lines it is linearly distributed with the density $\frac{1}{p_{k}}$. At that, $\mu$-measure of each square $P_{k, n}$ equals 1 and points $w_{k, n}$ are the mass centers of square $P_{k, n}$ w.r.t. measure $\mu$. By $\nu$ we denote the discrete measure with the unit masses at points $w_{k, n}$. It was shown in work [2] that under these conditions outside a small exceptional set $E$ the relation

$$
\int \ln \left|1-\frac{z}{w}\right| d(\nu(w)-\mu(w))=O(\ln (|z|)), \quad|z| \longrightarrow \infty,
$$

holds true. Thus, it is sufficient to prove the relations

$$
\int \ln \left|1-\frac{z}{w}\right| d(\nu(w)-\mu(w)) \leqslant O(N(|z|)), \quad|z| \longrightarrow \infty,
$$

and

$$
\int \ln \left|1-\frac{z}{w}\right|(d \nu(w)-\mu(w)) \geqslant-2 \ln |z|+O(N(|z|)), \quad|z| \longrightarrow \infty
$$

outside the set $\bigcup_{k, n} P_{k, n}^{q}$.

Indicating by $[x]$ the integer part of a number $x$, for each $p>0$ we have

$$
\begin{gathered}
\left|\frac{x}{p}-\left[\frac{x}{p}+\frac{1}{2}\right]\right| \leqslant \frac{1}{2}, x \in \mathbb{R}, \\
\left|\int_{0}^{x}\left(\frac{t}{p}-\left[\frac{t}{p}+\frac{1}{2}\right]\right) d t\right| \leqslant \frac{p}{8}, x \in \mathbb{R} .
\end{gathered}
$$

The restriction $\mu_{k}$ of measure $\mu$ on the critical line $\operatorname{Re} w=x_{k}+i t$ is generated by the function $\frac{t}{p_{k}}+C$, while the restriction of point measure $\nu$ is generated by the function $\left[\frac{t}{p_{k}}+\frac{1}{2}\right]+C$. We let

$$
\eta_{k}(t)=\left[\frac{t}{p_{k}}+\frac{1}{2}\right]-\frac{t}{p_{k}}, \quad L(z, w)=\ln \left|1-\frac{z}{w}\right|=\operatorname{Re} \ln \left(1-\frac{z}{w}\right) .
$$

By $\eta$ we denote the charge whose restriction on the vertical line $\operatorname{Re} w=x_{k}$ equals $\eta_{k}(t) d t$. Under these notation we need to estimate the integral

$$
\begin{aligned}
\int \ln \left|1-\frac{z}{w}\right| d(\nu(w)-\mu(w)) & =\sum_{k} \int_{-\infty}^{\infty} L\left(z, x_{k}+i t\right) d \eta_{k}(t) \\
& =-\sum_{k} \int_{-\infty}^{\infty} L^{\prime}\left(z, x_{k}+i t\right) \eta_{k}(t) d t=-\int L^{\prime}(z, w) d \eta(w) .
\end{aligned}
$$

We shall make use of the following representation $(w=s+i t)$

$$
L_{t}^{\prime}(z, w)=\operatorname{Re}\left(\frac{i}{w-z}-\frac{i}{w}\right)=-\operatorname{Im} \frac{z}{(w-z) w}=\operatorname{Im} \frac{1}{w}-\operatorname{Im} \frac{1}{w-z} .
$$

We fix a point $z=(x+i y) \in P_{n, j}$ assuming that $x, y \geqslant 0$, i.e., point $P_{n, j}$ lies in the first quadrant. We take a sufficiently small $\delta>0$ and introduce the squares: $Q(0)$ is the square centered at 0 with the sides parallel to the axes and of length $\delta r, r=|z|, Q(z)=Q(0)+z$. For sufficiently small $\delta$ squares are disjoint. Indeed, these squares lie in the circles of radius $\sqrt{2} \delta r$ 
with the same centers. Therefore, if $\delta<1 / \sqrt{8}$, the mentioned circles are disjoint. We shall suppose that $2 \delta<1 / \sqrt{8}$. In this case the distance between the squares obeys

$$
\operatorname{dist}(Q(0), Q(z))>\delta|z| \text {. }
$$

Lemma 2. Let $E$ be the exterior of two vertical strips with the bases $[-\delta r ; \delta r]$ (involving square $Q(0))$ and $[x-\delta r ; x+\delta r]$ (involving square $Q(z))$. Then $(w=s+i t)$

$$
\left|\int_{E} L_{t}^{\prime}(z, w) d \eta(w)\right|=O(1), \quad|z| \longrightarrow \infty .
$$

Proof. Let $x_{k} \notin[-\delta r ; \delta r] \bigcup[x-\delta r ; x+\delta r]$. We employ (2), (4) and Cauchy-Schwarz inequality

$$
\begin{aligned}
\left|\int_{-\infty}^{\infty} \eta_{k}(t) L^{\prime}\left(z, x_{k}+i t\right) d t\right| & \leqslant \frac{|z|}{2} \int_{-\infty}^{+\infty} \frac{1}{\left|x_{k}+i t\right|\left|x_{k}+i t-z\right|} d t \leqslant \\
& \leqslant \frac{|z|}{2}\left(\int_{-\infty}^{+\infty} \frac{d t}{\left|x_{k}+i t\right|^{2}}\right)^{\frac{1}{2}}\left(\int_{-\infty}^{+\infty} \frac{d t}{\left|x_{k}+i t-z\right|^{2}}\right)^{\frac{1}{2}}=\frac{\pi|z|}{2 \sqrt{\left|x_{k}\left(x-x_{k}\right)\right|}} .
\end{aligned}
$$

Lemma 1 (relation (1)) implies that for some constants $q<1, C>0$ and each $n, m \in \mathbb{Z}$, $n m>0,|m| \leqslant|n|$, the estimate

holds true. Hence,

$$
\frac{T_{m}}{T_{n}} \leqslant C q^{|n|-|m|}
$$

$$
\left|\frac{x_{m}}{x_{k}}\right| \leqslant C q^{|k|-|m|-2}
$$

Consider the indices $k$ obeying $x_{k} \geqslant x+\delta r$ and let $m>0$ be the smallest among them. Then

$$
\left|\sum_{k=m}^{\infty} \int_{-\infty}^{\infty} \eta_{k}(t) L^{\prime}\left(z, x_{k}+i t\right) d t\right| \leqslant \frac{\pi|z|}{2 \sqrt{\left|x_{m}\left(x-x_{m}\right)\right|}} \sum_{m}^{\infty} \sqrt{\left|\frac{x_{m}\left(x-x_{m}\right)}{x_{k}\left(x-x_{k}\right)}\right|} \leqslant \frac{\pi \sqrt{C}(3-\sqrt{q})}{4(1-\sqrt{q}) \delta} \prec 1 .
$$

In the same way we can estimate the sum of integrals w.r.t. the indices $k$ obeying $x_{k} \leqslant-\delta r$. And the same is true for the sum of integrals w.r.t. the indices $k$ for which $\delta r<x_{k} \leqslant x-\delta r$ if they exists. The proof is complete.

Lemma 3. Let $F=\mathbb{C} \backslash(E \bigcup Q(0) \bigcup Q(z))$. Then $(w=s+i t)$

$$
\left|\int_{F} L_{t}^{\prime}(z, w) d \eta(w)\right|=O(N(2|z|)), \quad|z| \longrightarrow \infty .
$$

Proof. The support of the charge $\eta=\nu-\mu$ on set $F$ is the union of the intervals $x_{k}+i(\delta r ;+\infty)$, $x_{k}+i(-\infty ;-\delta r ;), x_{k} \in(-\delta r ; \delta r), x_{k}+i(y+\delta r ;+\infty), x_{k}+(-\infty ; y-\delta r),\left|x-x_{k}\right| \leqslant \delta r$ and probably of bounded intervals $x_{k}+i(\delta r ; y-\delta r), x_{k} \in(x-\delta r ; \delta r)$.

Consider a bounded integral $(\delta r ; y-\delta r)$. By representation (4) and estimate (2) we have $\left(w=x_{k}+i t\right)$

$$
\left|\int_{\delta r}^{y-\delta r} \eta_{k}(t) L^{\prime}\left(z, x_{k}+i t\right) d t\right| \leqslant \frac{|z|}{2}\left|\int_{\delta r}^{y-\delta r} \frac{d t}{|w-z||w|}\right| \leqslant \frac{1}{2 \delta^{2}} .
$$

Consider an interval $x_{k}+i(\delta r ;+\infty)$. If $w=x_{k}+i t$ and $x_{k} \leqslant x-\delta|z|$, then $|w-z| \geqslant x-x_{k} \geqslant \delta|z|$, and thus $|w| \leqslant|w-z|+|z| \leqslant \frac{1+\delta}{\delta}|w-z|$. Hence,

$$
|w-z| \geqslant \frac{\delta}{1+\delta}|w|
$$

It yields for $w=x_{k}+i t, t \in(\delta r ;+\infty)$, the estimate

$$
\left|L_{t}^{\prime}\left(z, x_{k}+i t\right)\right| \prec \frac{|z|}{|w|^{2}}
$$


Thus,

$$
\left|\int_{\delta r}^{+\infty} \eta_{k}(t) L^{\prime}\left(z, x_{k}+i t\right) d t\right| \prec \frac{|z|}{2} \int_{\delta r}^{+\infty} \frac{d t}{x_{k}^{2}+t^{2}} \leqslant \frac{1}{2 \delta} .
$$

In the same way we prove that the integrals over unbounded intervals of other kind are bounded as well. If a vertical line $\operatorname{Re} w=x_{k}$ intersects with set $Q(0) \cup Q(z)$, then $x_{k} \in(-\delta r ;(1+\delta) r)$, and thus, the amount of such lines is at most $N(2 r)$. The proof is complete.

Proposition 1. Let $\zeta=a+i b \in \mathbb{C}, \sigma \in(0 ; 1)$. Then

1. For each number $d$ we have

$$
\left|\operatorname{Im} \int_{b-\sigma p_{k}}^{b+\sigma p_{k}} \frac{\left(\frac{t}{p_{k}}-d\right)}{\left(x_{k}+i t\right)-\zeta} d t\right| \leqslant 2 \sigma
$$

2. If $\sigma p_{k} \leqslant \delta r$, then

$$
\left|\operatorname{Im} \int_{b \pm \sigma p_{k}}^{b \pm \delta r} \frac{\eta_{k}(t) d t}{\left(x_{k}+i t\right)-\zeta}\right| \leqslant \frac{1}{2 \sigma}
$$

Proof. 1. Since

$$
\begin{aligned}
\operatorname{Im} \int_{b-\sigma p_{k}}^{b+\sigma p_{k}} \frac{\left(\frac{t}{p_{k}}-d\right)}{\left(x_{k}-a\right)+i(t-b)} d t & =\frac{1}{p_{k}} \operatorname{Im} \int_{-\sigma p_{k}}^{\sigma p_{k}} \frac{\left(\tau+b-d p_{k}\right) d \tau}{\left(x_{k}-a\right)+i \tau} \\
& =\frac{1}{p_{k}} \operatorname{Im} \int_{-\sigma p_{k}}^{\sigma p_{k}} \frac{\tau d \tau}{\left(x_{k}-a\right)+i \tau}+\frac{b-d p_{k}}{p_{k}} \operatorname{Im} \int_{-\sigma p_{k}}^{\sigma p_{k}} \frac{d \tau}{\left(x_{k}-a\right)+i \tau}
\end{aligned}
$$

and

$$
\operatorname{Im} \int_{-\sigma p_{k}}^{\sigma p_{k}} \frac{d \tau}{\left(x_{k}-a\right)+i \tau}=\int_{-\sigma p_{k}}^{\sigma p_{k}} \frac{-\tau d \tau}{\left(x_{k}-a\right)^{2}+\tau^{2}}=0
$$

then

$$
\left|\operatorname{Im} \int_{b-\sigma p_{k}}^{b+\sigma p_{k}} \frac{\eta_{k}(t) d t}{\left(x_{k}-a\right)+i(t-b)}\right|=\frac{1}{p_{k}}\left|\operatorname{Im} \int_{-\sigma p_{k}}^{\sigma p_{k}} \frac{\tau d \tau}{\left(x_{k}-a\right)+i \tau}\right|=\frac{1}{p_{k}} \int_{-\sigma p_{k}}^{\sigma p_{k}} \frac{\tau^{2} d \tau}{\left|x_{k}-a\right|^{2}+\tau^{2}} \leqslant 2 \sigma .
$$

2. Integrating by parts, we obtain

$$
\int_{b+\sigma p_{k}}^{b+\delta r} \frac{\eta_{k}(t) d t}{x_{k}+i t-\zeta}=\left(\int_{b+\sigma p_{k}}^{b+\delta r} \eta_{k}(\tau) d \tau\right) \frac{1}{x_{k}+i \delta r-a}+\int_{b+\sigma p_{k}}^{b+\delta r}\left(\int_{b+\sigma p_{k}}^{t} \eta_{k}(\tau) d \tau\right) \frac{i d t}{\left(x_{k}+i t-\zeta\right)^{2}}
$$

Therefore, by estimate (3),

$$
\left|\operatorname{Im} \int_{b+\sigma p_{k}}^{b+\delta r} \frac{\eta_{k}(t) d t}{x_{k}+i t-\zeta}\right| \leqslant \frac{p_{k}}{4 \delta r}+\frac{p_{k}}{4} \int_{b+\sigma p_{k}}^{\infty} \frac{d t}{\left(x_{k}-a\right)^{2}+(t-b)^{2}} \leqslant \frac{1}{4 \sigma}+\frac{p_{k}}{4 \sigma p_{k}} \leqslant \frac{1}{2 \sigma} .
$$

The proof is complete.

Lemma 4 . The relation $(w=s+i t)$

$$
\left|\int_{Q(0)} L_{t}^{\prime}(z, w) d \eta(w)\right|=O(N(|z|)), \quad|z| \longrightarrow \infty
$$

holds true.

Proof. By representation (4) and estimate (5) we see that for $w \in Q(0)$ the estimate $|w-z| \geqslant$ $\delta|z|$ holds and hence,

$$
\left|\int_{-\delta r}^{\delta r} \frac{\eta_{k}(t) d t}{\left(x_{k}+i t\right)-z}\right| \leqslant \frac{1}{\delta r} \cdot(2 \delta r)=2
$$


The number of $x_{k} \in(-\delta r ; \delta r)$ equals $N(\delta r)$, thus,

$$
\left|\int_{Q(0)} \frac{1}{w-z} d \eta(w)\right|=O(N(\delta|z|)), \quad|z| \longrightarrow \infty .
$$

We let $\sigma=\frac{1}{2}$ if $\frac{p_{k}}{2}<\delta r$ and $\sigma=\frac{\delta r}{p_{k}}$ if $\frac{p_{k}}{2} \geqslant \delta r$. We apply Proposition 1 for $\zeta=0, d=0$. In the first case we obtain

$$
\left|\operatorname{Im} \int_{-\delta r}^{\delta r} \frac{\eta_{k}(t) d t}{x_{k}+i t}\right| \leqslant\left|\operatorname{Im} \int_{-\frac{p_{k}}{2}}^{\frac{p_{k}}{2}} \frac{\frac{t}{p_{k}} d t}{x_{k}+i t}\right|+\left|\int_{\frac{p_{k}}{2} \leqslant|t|<\delta r} \operatorname{Im} \frac{\eta_{k}(t) d t}{x_{k}+i t}\right| \leqslant 3,
$$

while in the second case

Hence, in all the cases we have $\left(w=x_{k}+i t\right)$

$$
\left|\operatorname{Im} \int_{-\delta r}^{\delta r} \frac{\eta_{k}(t) d t}{x_{k}+i t}\right| \leqslant 1
$$

$$
\left|\operatorname{Im} \int_{-\delta r}^{\delta r} \frac{\eta_{k}(t) d t}{w}\right| \leqslant 3
$$

Together with relation (6) it completes the proof.

Lemma 5. The upper estimate $(w=s+i t)$

$$
-\operatorname{Im} \int_{Q(z)} L_{t}^{\prime}(z, w) d \eta(w)=O(N(|z|)), \quad|z| \longrightarrow \infty
$$

is valid.

Proof. We again employ representation (4). Since $|w| \geqslant|z|-|w-z| \geqslant(1-\delta)|z|$ for $w \in Q(z)$, we have

$$
\left|\operatorname{Im} \int_{y-\delta r}^{y+\delta r} \frac{\eta_{k}(t) d t}{x_{k}+i t}\right| \leqslant \frac{1}{(1-\delta) r} \cdot(2 \delta r)=\frac{2 \delta}{1-\delta} .
$$

The amount of $x_{k} \in(x-\delta r ; x+\delta r)$ does not exceed $N((1+\delta) r)$, thus,

$$
\left|\operatorname{Im} \int_{Q(z)} \frac{1}{w} d \eta(w)\right|=O(N(2|z|)), \quad|z| \longrightarrow \infty .
$$

We fix a number $\sigma_{0} \in\left(0 ; \frac{1}{4}\right)$ and we split the set of indices $k$ such that $x_{k} \in(x-\delta r ; x+\delta r)$ into two parts:

$J$ is the indices for which there exists a point $p_{k}\left(m+\frac{1}{2}\right)$ in the segment $\left[\left(y-\sigma_{0} p_{k}\right) ;\left(y+\sigma_{0} p_{k}\right)\right]$, where $m$ is an integer;

$J_{1}$ is the other indices.

1. For $k \in J_{1}$ we apply Proposition 1 taking $\sigma=\sigma_{0}$ if $\sigma_{0} p_{k}<\delta r$ and $\sigma=\frac{\delta r}{p_{k}}$ if $\sigma_{0} p_{k} \geqslant \delta r$. In the latter case we obtain

while in the former

$$
\left|\operatorname{Im} \int_{y-\delta r}^{y+\delta r} \frac{\eta_{k}(t) d t}{x_{k}+i t-z}\right| \leqslant 2 \sigma_{0}
$$

$\left|\operatorname{Im} \int_{y-\delta r}^{y+\delta r} \frac{\eta_{k}(t) d t}{x_{k}+i t-z}\right| \leqslant\left|\operatorname{Im} \int_{y-\sigma_{0} p_{k}}^{y+\sigma_{0} p_{k}} \frac{\left(m-\frac{t}{p_{k}}\right) d t}{x_{k}+i t-z}\right|+\left|\int_{\sigma_{0} p_{k} \leqslant|y-t|<\delta r} \operatorname{Im} \frac{\eta_{k}(t) d t}{x_{k}+i t-z}\right| \leqslant 2 \sigma_{0}+\frac{1}{\sigma_{0}}$.

Bearing in mind estimate (7), we obtain

$$
\left|\sum_{k \in J_{1}} \int_{y-\delta r}^{y+\delta r} L_{t}^{\prime}\left(x_{k}+i t, z\right) \eta_{k}(t) d t\right|=O(N(2|z|)), \quad|z| \longrightarrow \infty
$$


Thus,

$$
\int \ln \left|1-\frac{z}{w}\right| d(\nu(w)-\mu(w))=\sum_{k \in J} \operatorname{Im} \int_{y-\delta r}^{y+\delta r} \frac{\eta_{k}(t) d t}{x_{k}+i t-z}+O(N(2|z|)), \quad|z| \longrightarrow \infty .
$$

2. For each index $k \in J$ there exists an integer $m=m(k)$ such that $\left|y-p_{k}\left(m+\frac{1}{2}\right)\right| \leqslant \sigma_{0} p_{k}$. We introduce the notation $y_{k}^{*}:=p_{k}\left(m(k)+\frac{1}{2}\right)$. By the second item in Proposition 1, as $|z| \longrightarrow \infty$,

$$
\sum_{k \in J} \operatorname{Im} \int_{y-\delta r}^{y+\delta r} \frac{\eta_{k}(t) d t}{x_{k}+i t-z}=\sum_{k \in J} \operatorname{Im} \int_{y-\sigma_{0} p_{k}}^{y+\sigma_{0} p_{k}} \frac{\eta_{k}(t) d t}{x_{k}+i t-z}+O(N(2|z|)) .
$$

We note that if $k_{1}=\min \{k \in J\}, k_{2}=\max \{k \in J\}$, then

$$
\sum_{k=k_{1}}^{k_{2}-1} p_{k}=\sum_{k=k_{1}}^{k_{2}-1}\left(T_{k+1}-T_{k}\right)=T_{k_{2}}-T_{k_{1}} \leqslant(x+\delta r)-(x-\delta r)=2 \delta r,
$$

hence,

$$
p_{k} \leqslant 2 \delta r, k \in J
$$

We let $I_{k}:=\left(y-\frac{p_{k}}{2} ; y+\frac{p_{k}}{2}\right)$ if $p_{k} \leqslant 2 \delta r$ and $I_{k}:=(y-\delta r ; y+\delta r)$ otherwise. By the estimate for $p_{k}$ the latter situation can occur only once $k=k_{1}, k_{2}$ if these indices are in $J$. Thus, as $|z| \longrightarrow \infty$,

$$
\int \ln \left|1-\frac{z}{w}\right| d(\nu(w)-\mu(w))=\sum_{k \in J} \operatorname{Im} \int_{I_{k}} \frac{\eta_{k}(t) d t}{x_{k}+i t-z}+O(N(2|z|)) .
$$

It remains to estimate the integrals over intervals $I_{k}, k \in J$.

3. Let $k \in J, I_{k}=\left(a_{k} ; b_{k}\right)$ and for some integer $m$

$$
\eta_{k}(t)=\left\{\begin{aligned}
m-\frac{t}{p_{k}}, & a_{k}<t \leqslant y_{k}^{*}, \\
m+1-\frac{t}{p_{k}}, & y_{k}^{*}<t \leqslant b_{k}
\end{aligned}\right.
$$

Then

$$
\begin{aligned}
\operatorname{Im} \int_{I_{k}} \frac{\eta_{k}(t) d t}{x_{k}+i t-z} & =\operatorname{Im} \int_{a_{k}}^{y_{k}^{*}} \frac{\left(m-\frac{t}{p_{k}}\right) d t}{x_{k}+i t-z}+\operatorname{Im} \int_{y_{k}^{*}}^{b_{k}} \frac{\left(m+1-\frac{t}{p_{k}}\right) d t}{x_{k}+i t-z} \\
& =\operatorname{Im} \int_{a_{k}}^{b_{k}} \frac{\left(m-\frac{t}{p_{k}}\right) d t}{x_{k}+i t-z}+\int_{y_{k}^{*}}^{b_{k}} \frac{(y-t) d t}{\left(x_{k}-x\right)^{2}+(y-t)^{2}} d t
\end{aligned}
$$

We estimate the first integral by means of Proposition 1

$$
\left|\operatorname{Im} \int_{a_{k}}^{b_{k}} \frac{\left(m-\frac{t}{p_{k}}\right) d t}{x_{k}+i t-z} \leqslant 4\right| .
$$

The second integral can be calculated as

$$
\int_{y_{k}^{*}}^{b_{k}} \frac{(y-t) d t}{\left(x_{k}-x\right)^{2}+(y-t)^{2}} d t=-\ln \sqrt{\frac{\left(x_{k}-x\right)^{2}+\left(y-b_{k}\right)^{2}}{\left(x_{k}-x\right)^{2}+\left(y-y_{k}^{*}\right)^{2}}} .
$$

By the assumption for $k \in J, k \neq k_{1}, k_{2}$, we have $\left|y_{k}^{*}-y\right|<\sigma_{0} p_{k}<\frac{p_{k}}{4}$. Hence, $\left|b_{k}-y\right| \geqslant$ $\left|b_{k}-y_{k}^{*}\right|-\left|y_{k}^{*}-y\right| \geqslant \frac{p_{k}}{2}-\frac{p_{k}}{4}=\frac{p_{k}}{4}>\left|y_{k}^{*}-y\right|$, and therefore,

$$
\ln \sqrt{\frac{\left(x_{k}-x\right)^{2}+\left(y-b_{k}\right)^{2}}{\left(x_{k}-x\right)^{2}+\left(y-y_{k}^{*}\right)^{2}}} \geqslant 0 .
$$


Together with (9), (10) it yields

$$
\int \ln \left|1-\frac{z}{w}\right| d(\nu(w)-\mu(w)) \leqslant O(N(2|z|)), \quad|z| \longrightarrow \infty .
$$

The proof is complete.

To complete the proof of Theorem 1, it remains to obtain the lower estimate outside the set $\bigcup_{k, m} P_{k, m}^{q}$ under the assumptions that $z \in P_{n, j}$. By estimates (9), (10) we just need to show the lower estimate for the sum of integrals

$$
\sum_{k \in J} \int_{y_{k}^{*}}^{b_{k}} \frac{(y-t) d t}{\left(x_{k}-x\right)^{2}+(y-t)^{2}} d t=-\sum_{k \in J} \ln \sqrt{\frac{\left(x_{k}-x\right)^{2}+\left(y-b_{k}\right)^{2}}{\left(x_{k}-x\right)^{2}+\left(y-y_{k}^{*}\right)^{2}}} .
$$

We split the set of indices $J$ into three parts: $J_{0}=\left\{k \in J, 0 \leqslant x_{k}<x\right\}, J_{-}=\left\{k \in J, x_{k}<0\right\}$, $J_{+}=\left\{k \in J, x<x_{k}\right\}$. It is clear that

$$
\ln \sqrt{\frac{\left(x_{k}-x\right)^{2}+\left(y-b_{k}\right)^{2}}{\left(x_{k}-x\right)^{2}+\left(y-y_{k}^{*}\right)^{2}}} \leqslant \ln \sqrt{1+\frac{p_{k}^{2}}{4\left(x-x_{k}\right)^{2}}}
$$

Let $z \in P_{n, j}$, but $z \notin P_{n, j}^{q}$. If $k \in J_{0} k \neq n$, then $x-x_{k} \geqslant \frac{p_{k}}{2}$, and therefore,

$$
\ln \sqrt{\frac{\left(x_{k}-x\right)^{2}+\left(y-b_{k}\right)^{2}}{\left(x_{k}-x\right)^{2}+\left(y-y_{k}^{*}\right)^{2}}} \leqslant \ln \sqrt{2} .
$$

If $n \in J_{0}$ and $k=n$, by condition $z \notin P_{n, j}^{q}$ we have $x-x_{n} \leqslant \frac{q p_{k}}{2}$. Thus,

$$
\ln \sqrt{\frac{\left(x_{k}-x\right)^{2}+\left(y-b_{k}\right)^{2}}{\left(x_{k}-x\right)^{2}+\left(y-y_{k}^{*}\right)^{2}}} \leqslant \ln \sqrt{1+\frac{1}{q^{2}}} .
$$

Two latter estimates imply

$$
\sum_{k \in J_{0}} \operatorname{Im} \int_{I_{k}} \frac{\eta_{k}(t) d t}{x_{k}+i t-z}=O(N(2|z|)), \quad|z| \longrightarrow \infty .
$$

If $k \in J_{+}, k \neq n$, then $x-x_{k+1} \geqslant \frac{p_{k}}{2}$, and assuming $\left|x-x_{n}\right| \geqslant 1$, we obtain

$$
\sum_{k \in J_{+}} \ln \sqrt{\frac{\left(x_{k}-x\right)^{2}+\left(y-b_{k}\right)^{2}}{\left(x_{k}-x\right)^{2}+\left(y-y_{k}^{*}\right)^{2}}} \leqslant N(2 r)+\ln \prod_{k \in J_{+}} \frac{p_{k}}{2\left(x_{k}-x\right)}=N(2 r)+\ln \delta r .
$$

In the same way we get

$$
\sum_{k \in J_{-}} \ln \sqrt{\frac{\left(x_{k}-x\right)^{2}+\left(y-b_{k}\right)^{2}}{\left(x_{k}-x\right)^{2}+\left(y-y_{k}^{*}\right)^{2}}} \leqslant N(2 r)+\ln \prod_{k \in J_{-}} \frac{p_{k}}{2\left(x_{k}-x\right)}=1+\ln \delta r .
$$

Two latter inequalities and (11) follow that

$$
\sum_{k \in J} \ln \sqrt{\frac{\left(x_{k}-x\right)^{2}+\left(y-b_{k}\right)^{2}}{\left(x_{k}-x\right)^{2}+\left(y-y_{k}^{*}\right)^{2}}} \leqslant 2 \ln r+2 N(2 r) .
$$




\section{BIBLIOGRAPHY}

1. V.S. Azarin. On rays of completely regular growth of an entire function // Matem. Sbornik. 79(121):4(8), 463-476 (1969). [Math. USSR-Sbornik. 8:(4), 437-450 (1969).]

2. R.S. Yulmukhametov. Approximation of subharmonic functions // Anal. Math. 1985. 11:3, 257-282 (1985). (in Russian.)

3. Yu.I. Lyubarskii, M.L. Sodin. Analogues of sine like functions for convex domains. Preprtin no. 17, PTILT, Ukrainian AS, Kharkov (1986). (in Russian.)

4. Yu. Lyubarskii, E. Malinnikova On approximation of subharmonic functions // J. Anal. Math. 83:1, 121-149 (2001).

5. R.A. Bashmakov, A.A. Putintseva and R.S. Yulmukhametov. Entire functions of sine type and their applications // Alg. Anal. 22:5, 49-68 (2010). [St.-Petersburg Math. J. 22:5, 737-750 (2011).]

Konstantin Petrovich Isaev,

Institute of Mathematics CC USC RAS, Chernyshevskii str., 112,

450008, Ufa, Russia

E-mail: orbit81@list.ru

Rinad Salavatovich Yulmukhametov,

Institute of Mathematics CC USC RAS,

Chernyshevskii str., 112,

450008, Ufa, Russia

E-mail: Yulmukhametov@mail.ru

Artur Airatovich Yunusov,

Bashkir State University,

Zaki Validi str., 32,

450076, Ufa, Russia

E-mail: mc.yunusov@gmail.com 